\title{
The BDNF Val66Met Polymorphism Modulates the Generalization of Cued Fear Responses to a Novel Context
}

\author{
Andreas Mühlberger,',,2, Marta Andreatta', Heike Ewald', Evelyn Glotzbach-Schoon', Christian Tröger', \\ Christian Baumann ${ }^{1,3}$, Andreas Reif ${ }^{3}$, Jürgen Deckert ${ }^{3}$ and Paul Pauli' \\ 'Department of Psychology (Biological Psychology, Clinical Psychology, and Psychotherapy), University of Würzburg, Würzburg, Germany; \\ ${ }^{2}$ Department of Experimental Psychology (Clinical Psychology and Psychotherapy), University of Regensburg, Regensburg, Germany; \\ ${ }^{3}$ Department of Psychiatry, Psychosomatics, and Psychotherapy, University of Würzburg, Würzburg, Germany
}

\begin{abstract}
Brain-derived neurotrophic factor (BDNF) has a crucial role in activity-dependent synaptic plasticity and learning and memory. The human functional single-nucleotide BDNF rs6265 (Val66Met) polymorphism has been found to be associated with alteration in neural BDNF release and function correlating with altered emotional behavior. Here, we investigated for the first time the hypothesis that this polymorphism in humans modulates the context dependency of conditioned fear responses. Applying a new paradigm examining generalization of cued fear across contexts, 70 participants stratified for BDNF Val66Met polymorphism were guided through two virtual offices (context) in which briefly illuminated blue and yellow lights served as cues. In the fear context, one light (conditioned stimulus, CS +) but not the other light (CS - ) was associated with an electric shock (unconditioned stimulus, US). In the safety context, both lights were presented too, but no US was delivered. During the test phase, lights were presented again both in learning contexts and in a novel generalization context without any US. All participants showed clear fear conditioning to the CS + in the fear context as indicated by potentiation of startle responses and reports of fear. No fear reactions were found for the CS + in the safety context. Importantly, generalization of fear responses indicated by the potentiation of startle response to the CS + compared with the CS - in the novel context was evident only in the Met-carrying group. These are the first results to provide evidence in humans that BDNF modulates the generalization of fear responses. Such contextdependent generalization processes might predispose Met carriers for affective and anxiety disorders.

Neuropsychopharmacology (2014) 39, I I87-I 195; doi:I0.1038/npp.2013.320; published online I8 December 2013
\end{abstract}

Keywords: BDNF; startle reflex; cue conditioning; context conditioning; virtual reality

\section{INTRODUCTION}

Learning and memory are central mechanisms for adaption and survival. Whereas cue conditioning is thought to be a good research model for anxiety disorders characterized by phasic fear (eg, specific phobias), context conditioning is assumed to be relevant for anxiety disorders characterized by sustained anxiety (eg, panic disorder or post-traumatic stress disorder; Bouton et al, 2001; Craske et al, 2009; Davis et al, 2010; Mineka and Zinbarg, 2006). In addition, processes such as deteriorated extinction of fear memories and enhanced generalization of fear responses to other cues may contribute to the development and maintenance of anxiety disorders (Bouton et al, 2006; Dunsmoor et al, 2011; Lissek et al, 2005, 2010).

An important vulnerability factor believed to affect the acquisition and extinction of fear is the functionality of brain-derived neurotrophic factor (BDNF). BDNF, a mem-

*Correspondence: Professor A Mühlberger, Department of Experimental Psychology (Clinical Psychology and Psychotherapy), University of Regensburg, Universitätsstrasse 31, Regensburg 95053, Germany, Tel: +49 941 9436040, Fax: +49941 943816040,

E-mail: andreas.muehlberger@psychologie.uni-regensburg.de

Received I April 2013; revised 15 October 2013; accepted 16 October 2013; accepted article preview online 19 November 2013 ber of the neurotrophin family (Thoenen, 1995), is centrally involved in long-term potentiation (LTP) and adult synaptic plasticity (Bramham and Messaoudi, 2005; Korte et al, 1995; Tyler et al, 2002). Although BDNF is found throughout the entire brain, high concentrations have been observed mainly in regions involved in learning and memory (eg, hippocampus, amygdala, cerebral cortex, and cerebellum; Conner et al, 1997; Hofer et al, 1990). In humans, the single-nucleotide BDNF rs6265 (Val66Met) polymorphism leads to an exchange of amino acids from valine (Val) to methionine (Met) at codon 66 (Egan et al, 2003; Thoenen, 1995). In vitro studies investigating the functional effects of this polymorphism confirmed substantially less BDNF release in hetero- and homozygous BDNF Met-carrying cells than in homozygous BDNF Val cells (Chiaruttini et al, 2009; Egan et al, 2003). Thus, met carriers are likely characterized by a reduced BDNF synaptic functionality.

The importance of the BDNF Val66Met polymorphism for anxiety has been suggested by animal and human studies using associative learning paradigms. Two animal studies revealed an impaired context-dependent memory for BDNF + /Met and BDNF Met/Met mice compared with wild-type mice, whereas no effects for amygdala-dependent cue conditioning were found (Chen et al, 2006; Liu et al, 2004). These authors also found a reduced volume of the 
hippocampus due to an altered dendritic morphology of the gyrus dentatus (Chen et al, 2006) and thus suggest that the context-specific impairment may be due to reduced BDNF signaling in the hippocampus. Moreover, this alteration in the hippocampal anatomy was associated with enhanced anxiety-like behaviors. Because extinction learning is strongly context and hippocampus dependent (Bouton et al, 2006), effects of BDNF Val66Met polymorphism on extinction learning have to be expected too. Indeed, BDNF Met-carrying mice showed impaired extinction learning after aversive cue conditioning ( $\mathrm{Yu}$ et al, 2009). Moreover, fear extinction was impaired by hippocampus-specific deletion of BDNF (Heldt et al, 2007), and correlates with epigenetic regulation of the BDNF-encoding gene (Bredy et al, 2007).

Human studies have found mixed results. Whereas a study with a large sample found no modulation of cue conditioning by the Val66Met polymorphism (TorrentsRodas et al, 2012), two other studies reported reduced cue conditioning in Met-carrying participants (Hajcak et al, 2009; Lonsdorf et al, 2010). The discrepancies between these studies might be due to the different sample sizes of the homozygote Met/Met carriers in the studies $(n=3$ in both Hajcak et al, 2009 and Lonsdorf et al, 2010; and $n=10$ in Torrents-Rodas et al, 2012), or due to the different kinds of paradigms used (for further discussion, see Lonsdorf and Kalisch, 2011 and Torrents-Rodas et al, 2012). However, on the basis of these studies it remains unclear whether the Val66Met polymorphism of the human BDNF gene is specifically involved in hippocampus-dependent learning or rather mediates both hippocampus- and amygdala-dependent learning. Another study (Soliman et al, 2010) directly compared cued aversive conditioning and extinction in mice and humans and found further support for the role of the BDNF Val66Met polymorphism in context-dependent learning. Thus, BDNF polymorphism had no effect on cued conditioning, but on extinction where Met carriers responded with greater amygdala and less hippocampus as well as ventromedial prefrontal cortex (vmPFC) activation than homozygous for the Val allele.

Overall, there is convergent evidence from animal studies that BDNF Val66Met polymorphism is particularly involved in hippocampus-dependent learning such as the acquisition of contextual fear and the extinction of cued fear. Regarding fear generalization studies on the influence of BDNF are lacking, although generalization is actually discussed to contribute to pathological fear (Lissek, 2012) and has recently been found to be hippocampus dependent (Lissek et al, 2013). Only two human studies have investigated the influence of the BDNF polymorphism on generalization of conditioned fear over cues and found no reliable effect (Hajcak et al, 2009; Torrents-Rodas et al, 2012). Nevertheless, generalization of conditioned fear to novel contexts has to be expected because it is hippocampus dependent and may be a relevant mediator of pathological fear.

The present study examines in humans whether the BDNF Val66Met polymorphism modulates the interplay between contextual and cued fear conditioning. On the basis of the suggested association among BDNF, hippocampus, and context conditioning, we were particularly interested in investigating whether fear responses to a cue might be generalized in a novel context. To this end, we designed a differential aversive cue conditioning paradigm in which the meaning of a cue is clarified by the information entailed by the context. To this purpose, we made the cue conditioning dependent on context, ie, a cue predicted an unconditioned stimulus (US) in one specific context (fear context) but the same cue was not followed by an US in another context (save context). This design should lead to cue conditioning as well as background context conditioning (see Baas et al, 2004 and Phillips and LeDoux, 1992). Most importantly, during the following generalization phase, the conditioned stimuli were presented without the US in the fear context, the safety context, and a third novel context (the generalization context). Fear responses to the cues and the contexts were separately assessed using self-reported anxiety and startle responses (see Baas et al, 2004 and Phillips and LeDoux, 1992). We expected deteriorated background context conditioning, deteriorated extinction, and enhanced context-related generalization of aversive cue conditioning over contexts in Met compared with Val/Val carriers.

\section{MATERIALS AND METHODS}

\section{Participants}

Ninety-three healthy German volunteers were selected from a genetic data bank of 512 persons recruited from a genetic screening project (Collaborative Research Center SFB-TRR 58, project Z2). In a double-blind design, participants were chosen on the basis of their BDNF Val66Met polymorphism. The study was approved by the ethic board of the Medical Faculty of the University of Würzburg. All participants gave their informed consent and received $25 €$ for their participation.

Participants were ascertained to have an absence of axis I mental disorders by trained psychologists using the German version of the Mini International Neuropsychiatric Interview (MINI; Lecrubier et al, 1997). Participants suffering from a specific phobia were excluded only if their trait anxiety scores were high ( $T$-scores $\leqslant 60$; transformation performed within the gender and age groups of participants). On the base of this criterion, six participants (four homozygous Val carriers and two Met carriers) were excluded. Students of psychology were also excluded because of possible confounding factors due to their studies.

Nineteen participants (6 Val/Val, $2 \mathrm{Met} / \mathrm{Met}$, and $11 \mathrm{Val} /$ Met) were excluded from the analysis: 12 because of technical problems, 2 because they felt asleep, 2 because they interrupted the experiment, and 3 because the US electrodes dropped off during the experiment. Four additional participants ( $3 \mathrm{Val} / \mathrm{Val}$ and $1 \mathrm{Val} / \mathrm{Met}$ ) were excluded because of low startle amplitudes (overall mean amplitude below $5 \mu \mathrm{V}$ ). As a result, 35 participants carriers of at least $1 \mathrm{BDNF} 66 \mathrm{Met}$ allele (31 Val/Met and $4 \mathrm{Met} / \mathrm{Met}$; 23 women, mean age $=24.03$ years, $S D=3.68$; range: $21-40$ years; non-smokers: 31$)$ and 35 participants homozygous for the Val66 allele (23 women, mean age $=24.77$ years, $\mathrm{SD}=3.61$; range: 20-37 years; non-smokers: 30 ) were included in the analyses. Groups did not differ with regard to age, education, caffeine intake ( $n$. of cups of coffee per day, Val: $\mathrm{M}=1.04, \mathrm{SD}=0.97$; Met $+: \mathrm{M}=1.40, \mathrm{SD}=1.71$ ), smoking ( $\mathrm{n}$. of cigarettes per day, Val: $\mathrm{M}=1.36, \mathrm{SD}=3.99$; Met $+: \quad \mathrm{M}=0.52, \mathrm{SD}=1.85)$, or state anxiety (Val: $\mathrm{M}=35.38, \quad \mathrm{SD}=5.10 ; \mathrm{Met}+\mathrm{M}=35.46, \quad \mathrm{SD}=5.60)$ as assessed by the German version of the State-Trait Anxiety 
Inventory (STAI; Laux et al, 1981) (ps>0.27). We also found no group differences in the Wild-Intelligenz-Test (WIT-2; Kersting et al, 2008), which we included to test for spatial comprehension $(t(68)=0.93, p=0.358)$.

\section{Genotyping}

DNA was extracted from $18 \mathrm{ml}$ EDTA blood using a standard de-salting protocol. BDNF genotype was determined using a standard protocol (Hünnerkopf et al, 2007). Briefly, a 274-bp amplicon was amplified by PCR using a reaction mix containing $20 \mathrm{ng}$ of genomic DNA in $75 \mathrm{mM}$ Tris-HCl ( $\mathrm{pH}$ 9.0), $20 \mathrm{mM}$ ammonium sulfate, $0.01 \%$ Tween-20, $1.5 \mathrm{mM}$ magnesium chloride, $0.4 \mathrm{M}$ of each of the primers ( $5^{\prime}$-AAA GAA GCA AAC ATC CGA GGA CAA G and $5^{\prime}$-ATT CCT CCA GCA GAA AGA GAA GAG G), $0.4 \mathrm{mM}$ dNTP, and $1 \mathrm{U}$ Taq polymerase. After an initial denaturation step at $95{ }^{\circ} \mathrm{C}$ for $5 \mathrm{~min}, 35$ cycles of denaturation at $95^{\circ} \mathrm{C}$ for $30 \mathrm{~s}$, annealing at $55^{\circ} \mathrm{C}$ for $40 \mathrm{~s}$, and extension at $72{ }^{\circ} \mathrm{C}$ for $50 \mathrm{~s}$ were performed, followed by a final extension at $72{ }^{\circ} \mathrm{C}$ for $5 \mathrm{~min}$. PCR products were digested with NlaIII and visualized on an agarose gel.

\section{Stimulus Material}

The US was a 200-ms cutaneous electric pulse applied to the left forearm by a constant-current stimulator (DS7A, Digitimer, Letchworth Garden City, UK) at each individual's pain threshold, which was determined before the experiment (see Andreatta et al, 2010). Groups did not differ in pain threshold (Val/Val group: $2.61 \mathrm{~mA}, \mathrm{SD}=1.34$; Met-carrying group: $2.3 \mathrm{~mA}, \mathrm{SD}=0.90$, n.s.) or in US evaluation both before (Val/Val group: 5.31, $\mathrm{SD}=1.21$; Met-carrying group: $5.4, \mathrm{SD}=1.17$, n.s.) and after (Val/Val group: $4.94, \mathrm{SD}=1.44$; Met-carrying group: $4.5, \mathrm{SD}=1.69$, n.s.) the experiment.

Cues and contexts were created with the Source Engine (Valve Corporation, Bellevue, USA). Three virtual reality (VR) offices served as contexts (ie, a fear context, a safety context, and a generalization context; Glotzbach et al, 2012). The three offices differed in furniture and window views (skylines, mountains, and village). The choice of the three office rooms was based on the intention to use stimuli of the same category to exclude preexisting different emotional value of contexts (Dunsmoor et al, 2011; Lissek et al, 2010). The three rooms represented the fear, the safety, or the novel context (see procedure), counterbalanced among participants. Two colored lights (blue and yellow) switched on for $8 \mathrm{~s}$ while participants were within the contexts served as conditioned stimuli ( $\mathrm{CS}+$ and $\mathrm{CS}-$ ). Contexts and colored lights were counter balanced among participants. Notably, the choice of the three office rooms was due to the intent to use stimuli of the same category in parallel to previous generalization studies (Dunsmoor et al, 2011; Lissek et al, 2010).

The virtual environment was displayed by a Z800 3D Visor head-mounted display (HMD; eMagin, Hopewell Junction, USA). The head position was monitored with an electromagnetic tracking device (Patriot, Polhemus, Colchester, USA) to adapt the field of view. The software CyberSession (built in-house) was used to control the experimental paradigm.
A burst of white noise of $95 \mathrm{~dB}$ with a duration of $50 \mathrm{~ms}$ presented binaurally over headphones served as a blinkeliciting (startle) stimulus.

\section{Procedure}

After arrival in the laboratory, participants filled out the informed consent, electrodes were attached, and the pain threshold was assessed. Participants were instructed that they would be guided into several virtual offices where a lamp would turn on and off, and electrical stimuli as well as loud white noises would be also presented. Before beginning, three startle stimuli were delivered to decrease the initial startle reactivity. Afterwards, participants underwent a habituation phase, in which they freely explored the three contexts by means of a joystick for $2 \mathrm{~min}$ each. In each room, two additional habituation startle stimuli were delivered to decrease the initial reactivity of the startle response and reduce habituation during the experimental phases (see Grillon et al, 2006). The groups did not prefer one room more than the other one for their first $\left(\chi^{2}(2, N=70)=0.93\right.$, $p=0.629)$, second $\left(\chi^{2}(2, N=70)=1.14, p=0.566\right)$, or third $\left(\chi^{2}(2, N=70)=4.29, p=0.117\right)$ entrances.

During the acquisition phase, participants entered two out of three offices. A trial started in the middle of a corridor, the participant was then guided through the office for $2.5 \mathrm{~min}$ by a prerecorded path and ended with the exit from the office. Participants were always able to adapt their line of sight in the VR by head movements. Room entrance was pseudo-randomized, that is, the same room was not entered more than twice in a row. This phase consisted of four guided tours through the fear context, and four tours through the safety context. During each trial, a blue and a yellow light (CS + and $C S-$ ) was turn on three times, that is each light was presented 12 times in each context. The US was presented at CS + offset in the fear, but not in the safety context. In both contexts, the CS - was never associated with the US. The time between one light's offset and the next light's onset varied between 9 and $21 \mathrm{~s}$ (mean: $15 \mathrm{~s}$ ). To measure cue conditioning, six startle stimuli were presented during the $\mathrm{CS}+$ and six during the $\mathrm{CS}-$ in the fear context. The same was done in the safety context. Six additional startle stimuli were delivered in each room during periods when the lights were turned off to measure context conditioning. Altogether, 36 startle stimuli were delivered. The time between two startle probes varied between 15 and $30 \mathrm{~s}$. The minimum interval between the startle stimuli and the US was $10 \mathrm{~s}$ to avoid influence of the shock on the startle reaction (see Grillon et al, 2006).

The generalization test consisted of six trials lasting $2.5 \mathrm{~min}$ each. A trial started from the middle of the corridor, then participants were passively guided through the fear, the safety contexts, or a novel office (the generalization context), and ended with the exit from the room. Sequence of contexts was balanced across participants thus each context could have been entered as first. Each room was entered twice. The two lights were presented three times in each room, meaning six presentations of the CS + and six presentations of the $\mathrm{CS}-$ in each context. No US was delivered. Five startle stimuli were delivered during the $\mathrm{CS}+$ and five during the $\mathrm{CS}-$ in each context. Five additional startle stimuli were presented in each room when 
the lights were turned off to measure context conditioning. Altogether, 45 startle probes were delivered during the test.

Anxiety ratings of the lights and the offices were registered after the habituation phase, the acquisition phase, and the generalization test using a visual analogical scale (VAS) ranging from ' 0 ' (no anxiety at all) to ' 100 ' (high anxiety). No differences were detected in anxiety rating after the habituation phase ( $p s>0.193)$. In addition, we collected ratings for the valence and the arousal of the lights and the offices as well as participants' contingency ratings. These ratings are reported in the Supplementary Material (see Supplementary Table 2).

\section{Physiological Recording and Data Reduction}

The eye-blink component of the startle reflex was measured by electromyography (EMG) of the left orbicularis oculi muscle with one $5 \mathrm{~mm} \mathrm{Ag/AgCl}$ electrode placed under the pupil of the left eye and the second one at $\sim 1 \mathrm{~cm}$ laterally, and registered continuously with a V-Amp 16 (BrainProducts, Munich, Germany) at a sampling rate of $1000 \mathrm{~Hz}$. Startle data were first filtered using a $28-\mathrm{Hz}$ low cutoff filter and a $500-\mathrm{Hz}$ high cutoff filter. A moving average of $50 \mathrm{~ms}$ was applied, and then the myographic signal was rectified. The baseline for the EMG level was set $50 \mathrm{~ms}$ before startle stimulus' onset (Grillon et al, 2006). The peak amplitude of the blink reflex was defined as the maximum of the integrated response curve in the $20-\mathrm{ms}$ to 120 -ms time window following the onset of the startle stimulus relative to baseline. Responses to startle stimuli were scored automatically, but coding was controlled and revised manually by an assessor blind to group membership. Trials with excessive baseline shifts or movement artifacts were excluded. A minimum of two valid startles per condition was required for inclusion in the analysis. On average, we excluded $5.6 \%$ of startle responses for the $\mathrm{Val} / \mathrm{Val}$ and $7.2 \%$ for the Met-carriers group $(t(68)=1.14, p=0.257)$. To reduce the inter-individual variability, raw data were standardized using $z$-score and T-score conversions (Blumenthal et al, 2005) and then averaged for each condition (CS + and $\mathrm{CS}-$ ) in each context (fear, safety, and generalization contexts) separately for the acquisition phase and the generalization test. Additionally, skin conductance was assessed (for method and results, see Supplementary Material).

\section{Data Analysis}

Cue and context conditioning data were analyzed separately for the acquisition phase and the generalization test (Baas et al, 2004). Cue conditioning was analyzed with repeated measures ANOVAs with the between-subjects factor BDNF genotype (Val/Val, Met carrying) and the within-subject factors cue $(\mathrm{CS}+$ and $\mathrm{CS}-$ ) and context (acquisition phase: fear and safety; generalization test: fear, safety, and generalization). Responses to the contexts alone (ie, between CS presentations within a given context) were considered for context conditioning and analyzed with ANOVAs with the between-subjects factor BDNF genotype (Val/Val, Met carrying) and the within-subjects factor context (acquisition phase: fear and safety; generalization test: fear, safety, and generalization).

\section{RESULTS}

\section{Acquisition of Conditioned Responses to Cues}

Startle. Analyses revealed significant main effects of context $(\mathrm{F}(1,68)=4.18, p=0.045)$ and cue $(\mathrm{F}(1,68)=13.42$, $p<0.001)$ and a significant Cue $\times$ Context interaction $(\mathrm{F}(1,68)=10.03, p=0.002)$, but no effects involving the BDNF genotype (all $p s>0.44$ ). The significant Cue $\times$ Context interaction indicates successful fear conditioning in the fear context with significantly enhanced startle responses to the $\mathrm{CS}+$ compared with the CS $-(t(69)=4.64, p<0.001)$, but not in the safety context $(t(69)=0.28, p=0.780)$. An additional explorative analysis of $\mathrm{CS}+$ reactions over contexts confirmed higher startle responses to the CS + in the fear than in the safety context $(t(69)=3.54, p=0.001)$ (see Figure 1 and Supplementary Table 1).

Anxiety ratings. Analyses revealed significant main effects of context $(\mathrm{F}(1,68)=18.56, \quad p<0.001)$ and cue $(\mathrm{F}(1,68)=16.12, p<0.001)$, and a significant Cue $\times$ Context interaction $(\mathrm{F}(1,68)=22.57, p<0.001)$, but no effects involving the $\mathrm{BDNF}$ genotype (all $p s>0.32$ ). The anxiety ratings for the CS + were higher than for the $\mathrm{CS}-$ in the fear context $(25.60 \pm 27.16$ vs $12.90 \pm 17.92 ; t(69)=4.82, p<0.001)$ but not in the safety context $(12.43 \pm 17.64$ vs $10.07 \pm 16.09$, $t(69)=1.51, p=0.136)$. Additionally, as analyzed in an explorative analysis, anxiety ratings for the CS + were higher in the fear than in the safety context $(t(69)=5.06, p<0.001)$ (see Supplementary Table 1 separated for genotype).

\section{Discrimination of Contexts During Acquisition}

Startle. Analyses of the startle responses to the contexts alone (ie, between CS presentations within a given context)

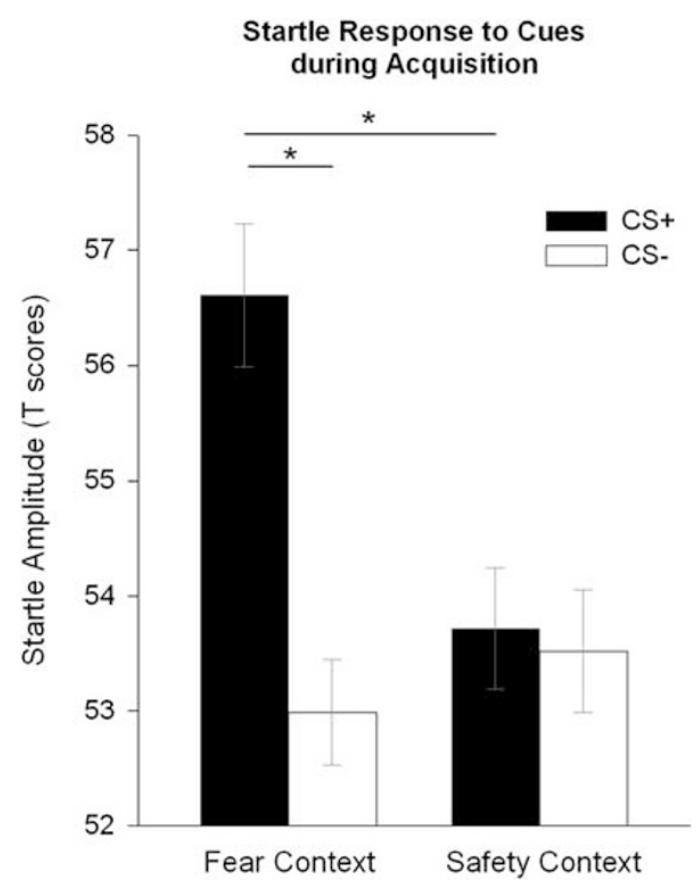

Figure I Startle responses to cues during acquisition. Black bars indicate the startle amplitude to the stimulus associated with the US in the fear but not in the safety context (CS + ) and white bars indicate the startle amplitude to the non-reinforced stimulus (CS - ). ${ }^{*} p<0.05$. 
Table I Conditioned Responses to Contexts for Genotypes

\begin{tabular}{|c|c|c|c|c|c|c|}
\hline & \multicolumn{2}{|c|}{ Fear CXT } & \multicolumn{2}{|c|}{ Safety CXT } & \multicolumn{2}{|c|}{ Novel CXT } \\
\hline & Val/Val & Met carriers & Val/Val & Met carriers & Val/Val & Met carriers \\
\hline Acquisition & | 3.43 (20.75) & | 4.43 (22.19) & $10.09(16.89)$ & |0.7| (|7.07) & - & - \\
\hline Generalization & $7.79(12.14)$ & $7.14(12.32)$ & $6.18(11.81)$ & $8.00(14.05)$ & $7.79(12.50)$ & $7.86(15.35)$ \\
\hline Acquisition & $53.23(4.29)$ & $52.42(4.07)$ & 50.61 (3.49) & $52.30(4.59)$ & - & - \\
\hline Generalization & $46.23(3.23)$ & $46.38(3.66)$ & $45.69(3.02)$ & $45.40(2.96)$ & $45.49(2.70)$ & $46.05(4.12)$ \\
\hline
\end{tabular}

Note: Means (standard deviations) of the anxiety ratings and the startle amplitudes (T scores) are reported separately for the Genotype groups. Conditioned responses to contexts were assessed when no cue was present.

revealed a main effect of context $(F(1,68)=4.42, p=0.039)$, but no main effect of BDNF genotype $(\mathrm{F}(1,68)=0.36$, $p=0.550)$ indicating an overall enhanced startle response in the fear compared with the safety context. On the basis of a marginally significant Context $\times \mathrm{BDNF}$ genotype interaction $(\mathrm{F}(1,68)=3.65, p=0.060)$, we exploratory examined context effects within the BDNF genotype groups. Enhanced responses in the fear compared with the safety context were found in the Val carriers $(\mathrm{F}(1,34)=8.91, p=0.005)$, but not in the Met carriers $(\mathrm{F}(1,34)=0.02, p=0.898)$. We performed the same analysis without considering the nine smokers in our sample. We found a significant main effect of context $(\mathrm{F}(1,57)=4.64, p=0.035)$ and a significant interaction Context $\times$ BDNF genotype $(\mathrm{F}(1,57)=4.48$, $p=0.039)$. Post hoc $t$-tests revealed that homozygous for the Val polymorphism showed potentiation of startle response to the fear referred to the safety context $(t(28)=3.16, \quad p=0.004)$, but not the Met carriers $(t(29)=0.03, p=0.979)$. These results might give a hint for some deteriorated context discrimination of the Met carriers (see Table 1 and Figure 2), even if these results should be confirmed in further studies.

Anxiety ratings. Descriptively, after the acquisition phase, participants rated the fear context as more anxiety inducing than the safety context, although the main effect of context just failed to reach significance $(\mathrm{F}(1,68)=3.77, p=0.056)$ (see Table 1). No further effects were significant $(p s>0.84)$.

\section{Generalization Test: Contextual Modulation of Cued Fear Responses}

Startle. Startle amplitudes to cues were modulated by the context and by the participants' BDNF genotype (Cue $\times$ Context $\times$ BDNF genotype interaction: $\mathrm{F}(2,136)=4.14$, $p=0.021$; see Figure 3). Follow-up ANOVAs within context conditions revealed the following: First, for the fear context, a significant main effect of cue $(\mathrm{F}(1,68)=5.19, p=0.026)$ with increased startle responses for the $\mathrm{CS}+$ compared with the CS - was found, but no main effect of BDNF genotype $(\mathrm{F}(1,68)=0.08, p=0.780)$ and no Cue $\times \mathrm{BDNF}$ genotype interaction $(\mathrm{F}(1,68)=0.56, p=0.584)$. Second, for the safety context, no significant effects were found (all $p s>0.16)$. Finally, for the novel context, a Cue $\times$ BDNF

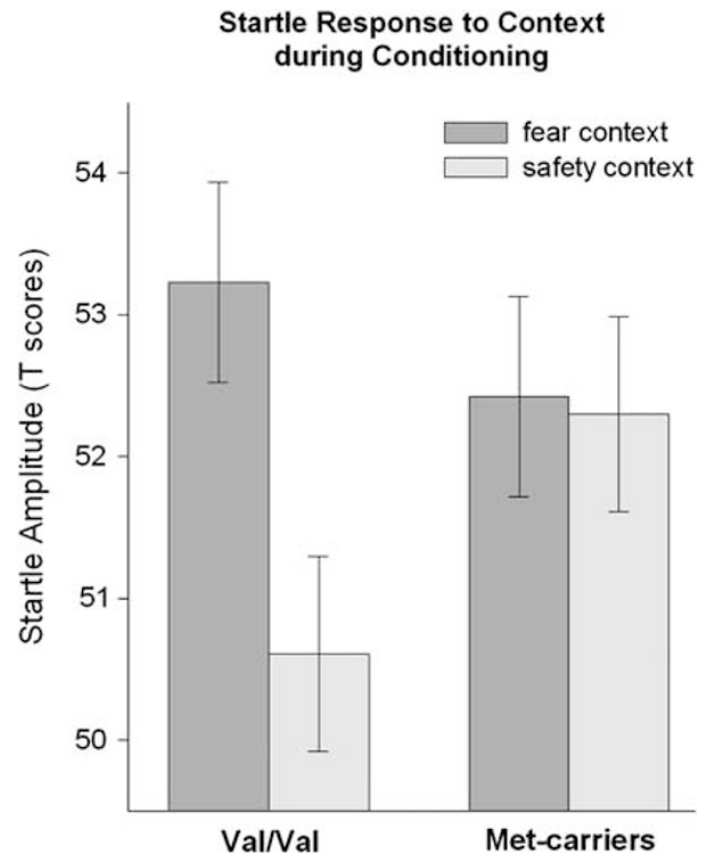

Figure 2 Startle responses to contexts during acquisition and during generalization test. Dark gray bars indicate the startle amplitude in the fear context, light gray bars represent the startle responses in the safety context, and stripped white bars represent the startle responses in the generalization context when no cue was presented. During acquisition, only the $\mathrm{Val} / \mathrm{Val}$ group showed contextual conditioning effects.

genotype interaction $(\mathrm{F}(1,68)=7.19, p=0.009)$ indicated that startle responses to the CS + compared with the CS were significantly increased in the Met-carrying group $(t(34)=2.67, p=0.012)$, but not in the Val group $(t(34)=1.22, p=0.232$ ) (see Figure 3 and Supplementary Table 1). Exploratory $t$-tests for the Met-carriers group indicated potentiated startle responses to the CS + in the fear context $(t(34)=3.40, p=0.002)$ and in the novel context $(t(34)=2.64, p=0.012)$ compared with the CS + in the safety context. No differences were revealed in startle responses to the CS - among the three contexts ( $p s>0.33)$. An exploratory comparison of startle response with the $\mathrm{CS}+$ in the novel context confirmed larger responses in the Met-carrying than in the Val group 


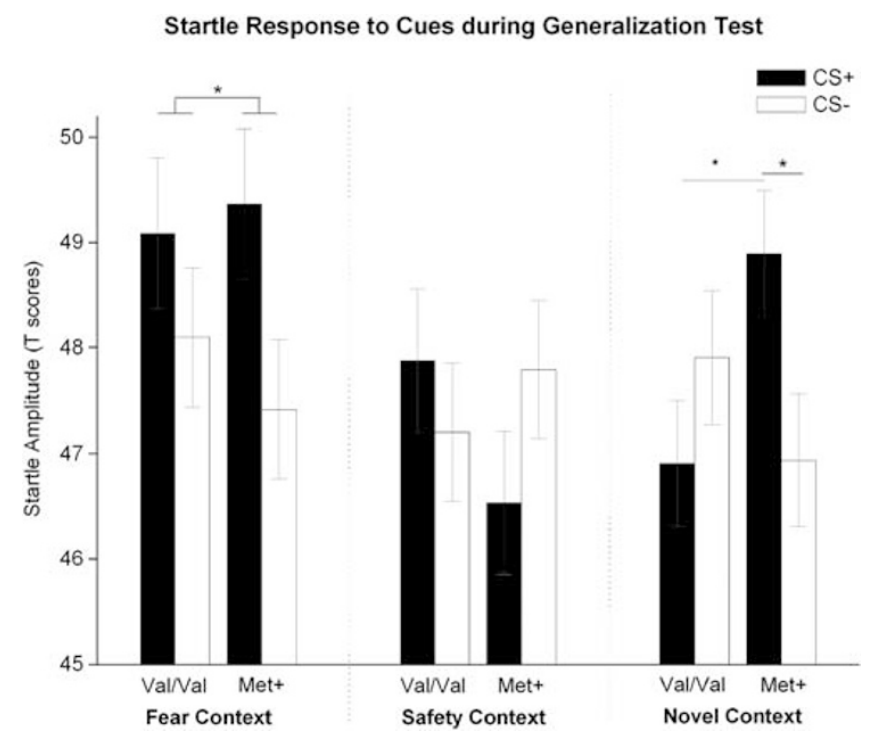

Figure 3 Startle responses during the generalization test. Black bars indicate the startle amplitude to the CS +, white bars represent the startle amplitude to the CS - presented separately for the fear, safety, and novel contexts. In the novel context, only the Met-carrying group reacted with an enhanced startle amplitude to the CS + , indicating a cue-conditioned fear generalization to novel contexts. ${ }^{*} p<0.05$.

$(t(68)=2.34, p=0.022)$. Thus, these results indicate generalization of cue conditioning to a novel context in the Met carriers only.

Anxiety ratings. The ANOVA indicated a significant main effect of cue $(\mathrm{F}(1,67)=14.14, p<0.001)$ and a significant Cue $\times$ Context interaction $(\mathrm{F}(2,134)=11.36, p<0.001)$, but no effects involving the BDNF genotype (all $p s>0.26$ ). Follow-up $t$-tests revealed that after the generalization test, the CS + still induced higher anxiety than the CS - in the fear context $(14.42 \pm 18.50$ vs $6.74 \pm 10.21, \quad t(68)=4.50$, $p<0.001)$ as well as in the safety context $(10.22 \pm 15.68 \mathrm{vs}$ $7.90 \pm 12.11, t(68)=2.20, p=0.031)$, but not in the novel context $\quad(10.80 \pm 17.77$ vs $8.99 \pm 14.08, \quad t(68)=1.69$, $p=0.096)$. Additional exploratory analyses indicate higher anxiety ratings for the $\mathrm{CS}+$ in the fear and in the novel context than the ratings for the CS + in the safety context $(14.42 \pm 18.50$ vs $10.22 \pm 15.68, t(68)=2.88, p=0.005$, and $14.42 \pm 18.50$ vs $10.80 \pm 17.77, t(68)=3.12, p=0.003$, respectively) (see Supplementary Table 1 separated for genotype).

\section{Context Effects During the Generalization Test}

No significant results were found for the startle responses or anxiety ratings assessed for the contexts alone (ie, between CS presentations within a given context) (all $p s>0.25)$ (see Table 1).

\section{DISCUSSION}

In this study, a new human paradigm examining generalization of cued fear across contexts was established using VR to create contexts (virtual rooms) and cues (lights within rooms). During the acquisition phase, differential conditioning to a $\mathrm{CS}+$ vs a $\mathrm{CS}-$ was implemented in the fear context, whereas the same cues were presented without consequences in the safety context. During the generalization test that followed, both cues were presented again in the fear context, the safety context, and a third novel context without any US presentation.

The developed experimental paradigm did work successfully as indicated by the following findings: First, contextmodulated differential cue conditioning as enhanced anxiety ratings and potentiated startle responses to the $\mathrm{CS}+$ compared with the CS - were apparent only in the fear but not in the safety context. Second, we observed context effects during acquisition as indicated by enhanced anxiety ratings and potentiated startle responses in the fear context compared with the safety context. Importantly, these context effects were measured when only the context, but no cue was presented. Third, during the generalization test, we observed lasting differential conditioning effects in the fear but not in the safety context. Finally, in the novel context we found no overall differential responses to the $\mathrm{CS}+$ compared with the $\mathrm{CS}-$, thus confirming a recent study with humans that revealed that aversive cue conditioning does not generalize to a novel context that was not presented during acquisition (Huff et al, 2011). We conclude that in humans cued fear responses can be learned specifically for the given acquisition context.

The main goal of our study was to evaluate the influence of the BDNF Val66Met polymorphism on context-dependent learning of cue-associated fear and safety (ambiguity) (Bouton, 2002) as well as the generalization of such learning to a novel context. First, as expected from animal studies (Chen et al, 2006; Liu et al, 2004), startle responses to the contexts alone (ie, in the absence of cues) suggest impaired background context-conditioning effects during acquisition in the Met-carrying participants; their startle data indicated on a trend level that they are less able to distinguish the fear from the safety context. Notably, US presentation was at the offset of CS + and although this is a common procedure in cue conditioning in humans, this might have caused a direct association between the US and the context to some degree (background conditioning). Considering that anxiety patients are less able to discriminate between danger and safety signals (Lissek et al, 2005) and that they generalize more their fear responses (Lissek et al, 2010), one might speculate that such 'direct' US-context association is affected by the BDNF Val66Met polymorphism. However, more participants should be tested to confirm this result.

Second and most important, we revealed with the generalization tests that Met carriers but not Val/Val participants generalize cued fear responses to the novel context; only Met carriers showed potentiated startle responses to the $\mathrm{CS}+$ compared with the $\mathrm{CS}-$ in the novel context. This effect was restricted to the novel context. Both Val/Val participants and Met carriers exhibited potentiated startle responses to the $\mathrm{CS}+$ compared with the $\mathrm{CS}$ - in the fear context and no startle potentiation to the $\mathrm{CS}+$ in the safety context. These effects cannot be explained by different reactions to the novel context between groups, since no differences between groups were found in reactions to contexts alone during the generalization test. We conclude that after establishing ambiguity of conditioning by making the cued fear signal context dependent during acquisition, Val/Val participants may 
generalize safety learning (no US after CS + in the safety context) to the novel context, whereas Met carriers may generalize fear learning (US after CS + in the fear context) to the novel context. This fundamental difference in the generalization after establishing the context-dependent ambiguity of a cue might contribute to the development of anxiety disorders because generalization of fear responses is evident in anxiety disorders, such as panic disorder, agoraphobia, and post-traumatic stress disorder (see Lissek, 2012). In the same vein, Met carriers in our study reacted to the CS + in the novel context as in the fear context.

We think that the Met-carrying group was less able to learn the association between context and conditioned fear than the $\mathrm{Val} / \mathrm{Val}$ group and therefore showed enhanced generalization of cued fear to the novel context. One might argue that our safety periods during acquisition served as extinction phases and consequently that our results could be explained by differences between groups in extinction learning, eg, insufficient extinction learning in Met carriers. However, the observed pattern of results cannot be explained by differences in extinction during the safety periods in the acquisition phase because of the following reasons: first, responses during the acquisition phase clearly indicate that participants learned to fear the CS+ specifically in the fear context. Simultaneously, no differences were found between CS + and CS - in the safety context. A similar context specificity effect has been seen in an earlier human conditioning study (Huff et al, 2011), thus confirming the validity of these findings. Importantly, we found no effect of genotype on these results, thus we have no hint for deficits in cue conditioning or extinction. Second, extinction deficits in Met carriers should have resulted in differences in response to the CS + compared with the CS - during the generalization test not only in the novel context, but also in the fear and the safety contexts. We did not find such differences. Further research should focus on the specific mechanisms of the genetic modulation of fear generalization across contexts and on the impact of this generalization on anxiety disorders.

We found no effects of the BDNF Val66Met polymorphism on aversive cue learning in the fear context, a finding that is in accordance with two animal studies (Chen et al, 2006; Liu et al, 2004) and one recent human study (Torrents-Rodas et al, 2012). By contrast, two human studies reported distinct influences of the BDNF Val66Met polymorphism on cue conditioning meaning that Met carriers showed impaired conditioning (Hajcak et al, 2009; Lonsdorf et al, 2010). In our study, descriptively and contrary to these results the CS +/CS - differentiation in the fear context seems to be enhanced; however, this effect is not confirmed statistically and therefore should not be interpreted. Moreover, differences in paradigms might also account for the inconsistencies in the results especially in cue conditioning. Since the Lonsdorf et al and Hajcak et al studies realized cue conditioning paradigms and we used a cue-across-context fear-generalization paradigm that established ambiguity of conditioning, the results could not be directly compared and the discrepancy in results might be due to this methodological difference. In contrast to the previous studies, our fear conditioning paradigm calls for a contextual differentiation of the fear expression, and thus we would suggest to have investigated hippocampusdependent learning.

Interestingly, the modulatory effect of the BDNF Val66Met polymorphism was evident only for startle responses, which can be considered as an implicit measure of fear (Andreatta et al, 2010), but not for explicit self-report data. This modulatory effect cannot attribute to the anxiety level of the participants, because the groups reported comparable anxiety at the beginning of the experiment. Additionally, we found no differential effects between groups for skin conductance (see Supplementary Material). This is not surprising because previous studies found skin conductance to be unaffected by genetic predispositions (Hajcak et al, 2009; Lonsdorf et al, 2010). However, our results confirm once more the power of the startle response as a crucial translational measure of emotional processing. Startle responses are directly modulated by emotional networks involving the amygdala (Lang et al, 2000) and more closely represent the activity of fear circuitries than self-report or skin-conductance data (Hamm and Weike, 2005). Because of these reasons and because rating data are more susceptible to demand and social influences, startle response may be considered as the most important measure in translational research on fear and anxiety (Davis et al, 2010).

According to previous findings, contextual fear learning is mediated by the hippocampus together with the amygdala. The BDNF gene mediates the traffic of the BDNF protein in the central nervous system and is implicated in the maintenance of the LTP in the hippocampus (Egan et al, 2003; Korte et al, 1995). LTP is the molecular mechanisms for synaptic plastic and therefore of learning and memory. Reduced hippocampal BDNF signaling was found to be associated with the BDNF Met genotype (Chen et al, 2006). One assumption is that our BDNF Val66Met effects on generalization depend on hippocampus BDNF activity, since the hippocampus mediates contextual learning. However, recent animal research also confirmed a modulatory effect of the BDNF Val66Met polymorphism on neural activity within the medial prefrontal cortex (Graybeal et al, 2011; Pattwell et al, 2012), and the prefrontal cortex is centrally involved in the control of emotional networks and the activity of the amygdala after conditioning (Klumpers et al, 2010). Therefore, the BDNF modulation of fear generalization across contexts has to be further examined to unravel the underlying neural mechanisms. One limitation of this study is that we presented the US with the offset of the CS + and the context was visible during the CS presentation, thus participants might associate the US not only with the CS + , but also with the context. Further experiments should consider presenting the CS during the $\mathrm{CS}+$ and/or fading out the context during CS presentation. Another limitation is the choice of a minimum of one third valid startle responses for a given condition only, ie, two out of six possible responses for the learning phase and two out of five possible responses for the generalization test. However, more strict criteria causing further exclusions would have limited the statistical power. For future studies, we recommend to increase the number of participants per group to be able to apply more restrictive exclusion criteria. Moreover, the sample under investigation consisted mainly of students having a mean age of 24 years, and it is well known that BNDF activity depends on age (Kennedy et al, 
2009). Thus, further studies should compare different age cohorts to search for differences in the modulation of BDNF on fear generalization across contexts. Furthermore, we did not investigate a group of patients who had an anxiety disorder. Thus, extrapolation of our results to the development or maintenance of anxiety disorders is speculative and has to be confirmed in further studies comparing healthy participants and patients with specific anxiety disorders (eg, agoraphobia).

Taken together, this is the first human study revealing evidence that the BDNF Val66Met polymorphism affects context conditioning as well as the generalization of cue conditioning to novel contexts after establishing contextdependent ambiguity. The enhanced fear generalization found in Met carriers might contribute to their vulnerability to emotional disorders (Davis et al, 2010). Further studies might identify the neural correlates (Pattwell et al, 2012), investigate age and gender effects (Bath et al, 2012), and may also include epigenetic aspects of the mediation of the BDNF Val66Met polymorphism for the development, maintenance, and therapy of emotional disorders (Boulle et al, 2011).

\section{FUNDING AND DISCLOSURE}

Paul Pauli and Andreas Mühlberger are shareholders of a commercial company that develops virtual environment research systems for empirical studies in the fields of psychology, psychiatry, and psychotherapy. Andreas Reif has received research grants from Astra Zeneca, which however are not related to the subject of the present paper. Jürgen Deckert has been an investigator in clinical trials and given educational lectures sponsored by several pharmaceutical companies with no relation though to the present topic.

\section{ACKNOWLEDGEMENTS}

We sincerely thank Mathias Müller for his technical support; Anna-Sophia Fritsch for her help in collecting data; and Michael Sendtner and Robert Blum for their most helpful comments on an earlier version of the article. This work is part of the dissertation of Heike Ewald. The work was supported by the German Research Foundation (DFG): DFG-RTG1253/1 and Collaborative Research Center 'Fear, Anxiety, Anxiety Disorders,' SFB-TRR 58 project B1 to PP and $\mathrm{AM}$ and $\mathrm{Z} 2$ to JD, AR, and PP.

\section{REFERENCES}

Andreatta M, Mühlberger A, Yarali A, Gerber B, Pauli P (2010). A rift between implicit and explicit conditioned valence in human pain relief learning. Proc $R$ Soc B Biol Sci 277: 2411-2416.

Baas JM, Nugent M, Lissek S, Pine DS, Grillon C (2004). Fear conditioning in virtual reality contexts: a new tool for the study of anxiety. Biol Psychiatry 55: 1056-1060.

Bath KG, Chuang J, Spencer-Segal JL, Amso D, Altemus M, McEwen BS et al (2012). Variant Brain-Derived Neurotrophic Factor (Valine66Methionine) polymorphism contributes to developmental and estrous stage-specific expression of anxietylike behavior in female mice. Biol Psychiatry 72: 499-504.
Blumenthal TD, Cuthbert BN, Filion DL, Hackley S, Lipp OV, Van Boxtel A (2005). Committee report: guidelines for human startle eyeblink electromyographic studies. Psychophysiology 42: 1-15.

Boulle F, van den Hove D, Jakob S, Rutten B, Hamon M, van Os J et al (2011). Epigenetic regulation of the BDNF gene: implications for psychiatric disorders. Mol Psychiatry 17: 584-596.

Bouton ME (2002). Context, ambiguity, and unlearning: Sources of relapse after behavioral extinction. Biol Psychiatry 52: 976-986.

Bouton ME, Mineka S, Barlow DH (2001). A modern learning theory perspective on the etiology of panic disorder. Psychol Rev 108: 4-32.

Bouton ME, Westbrook RF, Corcoran KA, Maren S (2006). Contextual and temporal modulation of extinction: Behavioral and biological mechanisms. Biol Psychiatry 60: 352-360.

Bramham CR, Messaoudi E (2005). BDNF function in adult synaptic plasticity: The synaptic consolidation hypothesis. Prog Neurobiol 76: 99-125.

Bredy TW, Wu H, Crego C, Zellhoefer J, Sun YE, Barad M (2007). Histone modifications around individual BDNF gene promoters in prefrontal cortex are associated with extinction of conditioned fear. Learn Mem 14: 268-276.

Chen Z-Y, Jing D, Bath KG, Ieraci A, Khan T, Siao C-J et al (2006). Genetic variant BDNF (Val66Met) polymorphism alters anxietyrelated behavior. Science 314: 140-143.

Chiaruttini C, Vicario A, Li Z, Baj G, Braiuca P, Wu Y et al (2009). Dendritic trafficking of BDNF mRNA is mediated by translin and blocked by the G196A (Val66Met) mutation. Proc Natl Acad Sci USA 106: 16481-16486.

Conner JM, Lauterborn JC, Yan Q, Gall CM, Varon S (1997). Distribution of Brain-Derived Neurotrophic Factor (BDNF) protein and mRNA in the normal adult rat CNS: Evidence for anterograde axonal transport. J Neurosci 17: 2295-2313.

Craske MG, Rauch SL, Ursano R, Prenoveau J, Pine DS, Zinbarg RE (2009). What is an anxiety disorder? Depress Anxiety 26: 1066-1085.

Davis M, Walker DL, Miles L, Grillon C (2010). Phasic vs sustained fear in rats and humans: role of the extended amygdala in fear $v s$ anxiety. Neuropsychopharmacology 35: 105-135.

Dunsmoor JE, Prince SE, Murty VP, Kragel PA, LaBar KS (2011). Neurobehavioral mechanisms of human fear generalization. Neuroimage 55: 1878-1888.

Egan MF, Kojima M, Callicott JH, Goldberg TE, Kolachana BS, Bertolino A et al (2003). The BDNF val66met polymorphism affects activity-dependent secretion of BDNF and human memory and hippocampal function. Cell 112: 257-269.

Glotzbach E, Ewald H, Andreatta M, Pauli P, Mühlberger A (2012). Contextual fear conditioning effects predict subsequent avoidance behavior. Cogn Emot 26: 1256-1272.

Graybeal C, Feyder M, Schulman E, Saksida LM, Bussey TJ, Brigman JL et al (2011). Paradoxical reversal learning enhancement by stress or prefrontal cortical damage: Rescue with BDNF. Nat Neurosci 14: 1507-1509.

Grillon C, Baas JM, Cornwell B, Johnson L (2006). Context conditioning and behavioral avoidance in a virtual reality environment: Effect of predictability. Biol Psychiatry 60: 752-759.

Hajcak G, Castille C, Olvet DM, Dunning JP, Roohi J, Hatchwell E (2009). Genetic variation in brain-derived neurotrophic factor and human fear conditioning. Genes Brain Behav 8: 80-85.

Hamm AO, Weike AI (2005). The neuropsychology of fear learning and fear regulation. Int J Psychophysiol 57: 5-14.

Heldt SA, Stanek L, Chhatwal JP, Ressler KJ (2007). Hippocampusspecific deletion of BDNF in adult mice impairs spatial memory and extinction of aversive memories. Mol Psychiatry 12: 656-670.

Hofer M, Pagliusi SR, Hohn A, Leibrock J, Barde YA (1990). Regional distribution of brain-derived neurotrophic factor messenger-RNA in the adult-mouse brain. EMBO J 9: 2459-2464. 
Huff N, Alba Hernandez J, Fecteau M, Zielinski D, Brady R, LaBar KS (2011). Revealing context-specific conditioned fear memories with full immersion virtual reality. Front Behav Neurosci 5: 75.

Hünnerkopf R, Strobel A, Gutknecht L, Brocke B, Lesch KP (2007). Interaction between BDNF Val66Met and dopamine transporter gene variation influences anxiety-related traits. Neuropsychopharmacology 32: 2552-2560.

Kennedy KM, Rodrigue KM, Land SJ, Raz N (2009). BDNF val66met polymorphism influences age differences in microstructure of the corpus callosum. Front Hum Neurosci 3: 19.

Kersting M, Althoff K, Jäger AO (2008). Testmanual/Verfahrenshinweise zum WIT 2 (Wilde-Intelligenztest). Hogrefe: Göttingen.

Klumpers F, Raemaekers MAHL, Ruigrok ANV, Hermans EJ, Kenemans JL, Baas JMP (2010). Prefrontal mechanisms of fear reduction after threat offset. Biol Psychiatry 68: 1031-1038.

Korte M, Carroll P, Wolf E, Brem G, Thoenen H, Bonhoeffer T (1995). Hippocampal long-term potentiation is impaired in mice lacking brain-derived neurotrophic factor. Proc Natl Acad Sci USA 92: 8856-8860.

Lang PJ, Davis M, Ohman A (2000). Fear and anxiety: animal models and human cognitive psychophysiology. J Affect Disord 61: 137-159.

Laux L, Glanzmann P, Schaffner P, Spielberger CD (1981). Das State-Trait Angstinventar. Beltz Test: Weinheim.

Lecrubier Y, Sheehan DV, Weiller E, Amorim P, Bonora I, Harnett Sheehan $\mathrm{K}$ et al (1997). The Mini International Neuropsychiatric Interview (MINI). A short diagnostic structured interview: reliability and validity according to the CIDI. Eur Psychiatry 12: $224-231$

Lissek S (2012). Toward an account of clinical anxiety predicated on basic neurally mapped mechanisms of Pavlovian fearlearning: the case for conditioned overgeneralization. Depress Anxiety 29: 257-263.

Lissek S, Bradford DE, Alvarez RP, Burton P, Espensen Sturges T, Reynolds RC et al (2013). Neural substrates of classically conditioned fear-generalization in humans: a parametric fMRI study. Soc Cogn Affect Neurosci.

Lissek S, Powers AS, McClure EB, Phelps EA, Woldehawariat G, Grillon $C$ et al (2005). Classical fear conditioning in the anxiety disorders: a meta-analysis. Behav Res Ther 43: 1391-1424.
Lissek S, Rabin S, Heller RE, Lukenbaugh D, Geraci M, Pine DS et al (2010). Overgeneralization of conditioned fear as a pathogenic marker of panic disorder. Am J Psychiatry 167: 47-55.

Liu IYC, Lyons WE, Mamounas LA, Thompson RF (2004). Brainderived neurotrophic factor plays a critical role in contextual fear conditioning. J Neurosci 24: 7958-7963.

Lonsdorf TB, Kalisch R (2011). A review on experimental and clinical genetic associations studies on fear conditioning, extinction and cognitive-behavioral treatment. Transl Psychiatry 1: e41.

Lonsdorf TB, Weike AI, Golkar A, Schalling M, Hamm AO, Ohman A (2010). Amygdala-dependent fear conditioning in humans is modulated by the BDNFval66met polymorphism. Behav Neurosci 124: 9-15.

Mineka S, Zinbarg R (2006). A contemporary learning theory perspective on the etiology of anxiety disorders-It's not what you thought it was. Am Psychol 61: 10-26.

Pattwell SS, Bath KG, Perez-Castro R, Lee FS, Chao MV, Ninan I (2012). The BDNF Val66Met polymorphism impairs synaptic transmission and plasticity in the infralimbic medial prefrontal cortex. J Neurosci 32: 2410-2421.

Phillips RG, LeDoux JE (1992). Differential contribution of amygdala and hippocampus to cued and contextual fear conditioning. Behav Neurosci 106: 274-285.

Soliman F, Glatt CE, Bath KG, Levita L, Jones RM, Pattwell SS et al (2010). A genetic variant BDNF polymorphism alters extinction learning in both mouse and human. Science 327: 863-866.

Thoenen H (1995). Neurotrophins and neuronal plasticity. Science 270: 593-598.

Torrents-Rodas D, Fullana MA, Arias B, Bonillo A, Caseras X, Andion $\mathrm{O}$ et al (2012). Acquisition and generalization of fear conditioning are not modulated by the BDNF-val66met polymorphism in humans. Psychophysiology 49: 713-719.

Tyler WJ, Alonso M, Bramham CR, Pozzo-Miller LD (2002). From acquisition to consolidation: on the role of brain-derived neurotrophic factor signaling in hippocampal-dependent learning. Learn Mem 9: 224-237.

Yu H, Wang Y, Pattwell S, Jing D, Liu T, Zhang Y et al (2009). Variant BDNF Val66Met polymorphism affects extinction of conditioned aversive memory. J Neurosci 29: 4056-4064.

Supplementary Information accompanies the paper on the Neuropsychopharmacology website (http://www.nature.com/npp) 